\section{pH en saliva total en pacientes con enfermedad periodontal del Servicio de Periodoncia de la Facultad de Odontología de la UNMSM}

Total saliva $\mathrm{pH}$ in patients with periodontal disease of UNMSM Dental Faculty Periodontal Service

\section{Resumen}

El presente estudio evalúa la relación entre el $\mathrm{pH}$ salival y la enfermedad periodontal. Se evaluaron 60 pacientes que acudieron a la clínica de pregrado de la Facultad de Odontología de la UNMSM (30 pacientes con gingivitis y 30 pacientes con periodontitis). Asimismo, se contó con un grupo control de 20 estudiantes de odontología periodontalmente sanos. Se evaluó el estado periodontal y se tomó muestras de saliva antes y después de realizarse el tratamiento de fase I. Se encontró que a diferencia de los pacientes del grupo control que presentaron un $\mathrm{pH}$ salival promedio de 6,9; los pacientes con gingivitis y periodontitis presentaron valores de 7,3 y 7,9 respectivamente. Después del tratamiento los pacientes que respondieron positivamente presentaron una disminución en los niveles de $\mathrm{pH}$ salival en relación al grupo control que se mantuvo. Los pacientes con gingivitis presentaron una reducción promedio de 0,3 (de 7,3 a 7,0). En el grupo de pacientes con periodontitis el $\mathrm{pH}$ se redujo de 7,9. a 7,3. El estudio concluyó que el $\mathrm{pH}$ salival presenta valores mayores en pacientes con enfermedad periodontal y se reduce luego de realizarse un tratamiento periodontal adecuado.

\begin{abstract}
The present study evaluates the relationship between salivary $\mathrm{pH}$ and periodontal disease. Sixty patients (30 gingivitis patients and 30 periodontitis patients) who went to UNMSM Dental School were evaluated. Also, we took a control group that included 20 dental students with healthy periodontum. We evaluated periodontal status and salivary samples were taken before and after phase I treatment. It was found a difference between control group that presented an average $\mathrm{pH}$ of 6.9 and gingivitis and periodontitis groups that showed values of 7.3 and 7.9 respectively. After treatment, patients who had a positive evolution presented a decrease in their salivary $\mathrm{pH}$ levels in comparison with control group that maintained its initial value. Gingivitis patients presented an average decrease of 0.3 (7.3 to 7.0). In periodontitis group patients, $\mathrm{pH}$ decreased from 7.9 to 7.3. This study concludes that salivary $\mathrm{pH}$ shows higher values in patients with periodontal disease and it decreases after the realization of an appropriate periodontal treatment.
\end{abstract}

\section{Sixto García Linares', Francis Bravo Castañola ${ }^{2}$, Jocelyn Ayala Luis ${ }^{3}$, Gua- dalupe Bardales Cuzquén ${ }^{3}$}

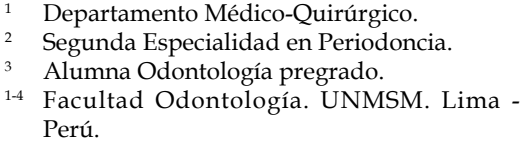

Correspondencia:

Esp. Sixto García Linares

Av. Brasil 2481-B Of. 205 Jesús María. Lima - Perú

Tlf: 6197000 / 3403

Sgarcial@unmsm.edu.pe

Palabras Clave: Enfermedad periodontal, $\mathrm{pH}$, saliva.

Key words: Periodontal disease, $\mathrm{pH}$, saliva.

\section{Introducción}

En los últimos años el entendimiento, diagnóstico y tratamiento de la enfermedad periodontal ha avanzada notablemente. Es así que muchas investigaciones relacionadas con la epidemiología, fisiopatología y bioquímica de la enfermedad periodontal han alterado grandemente en la visión actual de dicha patología.

Actualmente se conocen de muchos aspectos bioquímicas que se relacionan con la fisiopatología de la enfermedad periodontal. A través de las investigaciones, se está enfatizando y comprendiendo mejor el rol que cumplen en la aparición y progreso de la patología periodontal. Es así que se disponen de muchos valores para el fluido crevicular en pacientes con enfermedad periodontal. Sin embargo, existen escasos datos del papel en la patología periodontal de la saliva, siendo ésta el lugar dónde drena el fluido crevicular y sus diversos componentes. De este modo es factible creer que en la enfermedad periodontal el fluido crevicular modificada muchas propiedades de la saliva tales como su composición y $\mathrm{pH}$.

\section{Materiales y métodos}

El estudio se realizó en pacientes que acudieron a la clínica de pre grado de Periodoncia de la Facultad de Odontología de la Universidad Nacional Mayor de San Marcos. Se selecciono una muestra de 60 pacientes. De esa cantidad de pacientes 30 con diagnóstico inicial de gingivitis constituyeron el grupo de estudio 1 y los otros 30 con diagnóstico de periodontitis se 
incluyeron en el grupo de estudio 2 . Además se seleccionó una muestra de 20 pacientes adultos sin enfermedad periodontal que conformaron el grupo control.

Para ser divididos en los grupos de estudio de la patología periodontal determinada se utilizó el examen periodontal básico (EPB) propuesto por Lindhe. Es así que se constituyeron los grupos de pacientes con gingivitis y otro con pacientes con periodontitis. De acuerdo a este índice cada grupo fue divido en dos subgrupos según la severidad de la afección. El grupo de pacientes con gingivitis se dividió en gingivitis leve y gingivitis leve a moderada. El otro grupo de estudio correspondiente a los pacientes con periodontitis se dividió en pacientes con periodontitis leve y otro con periodontitis severa. Los individuos del grupo control fueron seleccionados al azar de entre estudiantes de Odontología cuyas edades oscilaron entres 20 y 40 años.

Cada paciente de los grupos de estudio fue sometido a tratamiento periodontal de Fase I (fisioterapia oral, instrucción para control de placa, raspaje supra e infragingival y alisado radicular) por parte de su operador. Los investigadores sólo realizaron las evaluaciones respectivas (examen periodontal y análisis de $\mathrm{pH}$ salival) antes y después del tratamiento.

En los tres grupos la toma de muestra salival y el examen periodontal se realizaban la cita siguiente a la primera consulta del paciente en la clínica de Periodoncia de la Facultad de Odontología de la UNMSM. Todas la muestras salivales iniciales se tomaron antes de cualquier procedimiento clínico de raspado o alisado radicular, fisioterapia o profilaxis.

Para el análisis de la muestra salival obtenida se utilizó un Potenciómetro modelo pHmeter 28 con electrodos Radiometer modelos Escalomel k401 y Gals g202c. Se utilizaron dos buffer de $\mathrm{pH} 7$ y 10 para el análisis espectrofotométrico de las muestras.

\section{Resultados}

En la medición del $\mathrm{pH}$ salival antes del tratamiento se encontró que el grupo control presentaba un $\mathrm{pH}$ promedio de 6,9. Los grupos de pacientes con gingivitis y periodontitis presentaron valores promedios de 7,3 y 7,9 respectivamente (Gráfico $N^{\circ} 1$ ).

Tabla $N^{0} 1$ Prueba t de student

\begin{tabular}{|c|c|c|c|c|}
\hline Grupos & $\begin{array}{c}\text { Respuesta al } \\
\text { tratamiento }\end{array}$ & Momento de la muestra & $\begin{array}{l}\text { Corre- } \\
\text { lación }\end{array}$ & $\begin{array}{l}\text { Sig (bi- } \\
\text { lateral) }\end{array}$ \\
\hline \multirow{4}{*}{$\begin{array}{l}\text { Grupo gin- } \\
\text { givitis con }\end{array}$} & \multirow{2}{*}{$\mathrm{Si}$} & Antes de tratamiento & \multirow{2}{*}{0,492} & \multirow{2}{*}{0,000} \\
\hline & & Después de tratamiento & & \\
\hline & \multirow{2}{*}{ No } & Antes de tratamiento & \multirow{2}{*}{0,700} & \multirow{2}{*}{0,024} \\
\hline & & Después de tratamiento & & \\
\hline \multirow{4}{*}{$\begin{array}{l}\text { Grupo peri- } \\
\text { odonitis }\end{array}$} & \multirow{2}{*}{$\mathrm{Si}$} & Antes de tratamiento & \multirow{2}{*}{0,447} & \multirow{2}{*}{0,000} \\
\hline & & Después de tratamiento & & \\
\hline & \multirow{2}{*}{ No } & Antes de tratamiento & \multirow{2}{*}{0,835} & \multirow{2}{*}{0,010} \\
\hline & & Después de tratamiento & & \\
\hline
\end{tabular}

Gráfico $\mathrm{N}^{\circ}$ 1: Niveles de $\mathrm{pH}$ en saliva total antes del tratamiento y estado periodontal

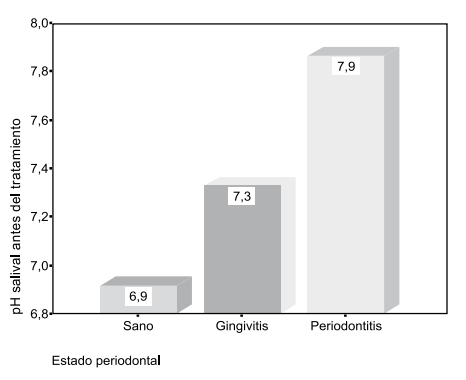

Gráfico $\mathrm{N}^{\circ}$ 2: Comparación del pH salival en pacientes sanos, con gingivitis y periodontitis que respondieron antes y después del tratamiento

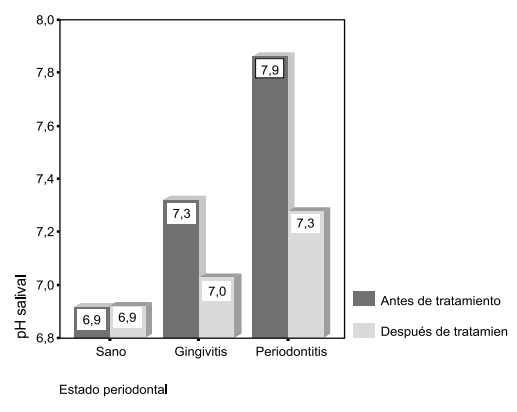

Gráfico N 3: Comparación del porcentaje de piezas afectadas en pacientes con gingivitis y periodontitis y el $\mathrm{pH}$ salival

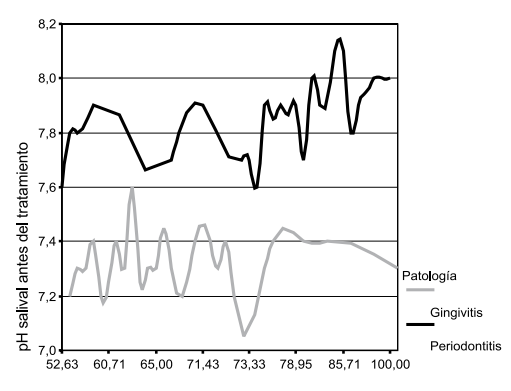

A medida que la severidad de la afección aumentaba, los niveles de $\mathrm{pH}$ salival se alcalinizaban. En los pacientes con gingivitis leve y moderada los valores de $\mathrm{pH}$ obtenidos fueron de 7,3 y 7,4 respectivamente. Los grupos de pacientes con periodontitis leve y severa presentaron niveles de 7,8 y 7,9.

Luego de 2 meses y habiendo realizado el tratamiento de fase I en todos los pacientes del estudio, se reevaluó el estado periodontal y tomó nuevas muestras de saliva. De los 60 pacientes tratados 20 pacientes no respondieron satisfactoriamente a la fase I (10 de cada grupo de estudio).

En los pacientes que respondieron positivamente al tratamiento, se observó una considerable reducción del $\mathrm{pH}$ salival. Los pacientes con gingivitis pasaron de un $\mathrm{pH}$ promedio de 7,3 a 7,0 . Mientras tanto, en el grupo con periodontitis se produjo una reducción de 0,6 (de 7,9 a 7,3). En el grupo control el pH se mantuvo constante a 6,9 en ambos momentos del estudio (Gráfico No 2).

En los pacientes que recibieron tratamiento de fase I, pero que no respondieron adecuadamente al tratamiento; el $\mathrm{pH}$ también se redujo pero en mucho menor. En los pacientes con gingivitis no hubo cambios manteniendo se el pH salival en 7,3. En el grupo de pacientes con periodontitis el $\mathrm{pH}$ se redujo sólo en 0,1 .

También se evalúo la relación entra la cantidad de piezas afectadas y su relación con la enfermedad periodontal. En los pacientes con gingivitis no se encontró una relación clara entre el nivel de $\mathrm{pH}$ salival y la cantidad de piezas afectadas. En el grupo con periodontitis si se observó que el $\mathrm{pH}$ salival aumentaba a medida que el porcentaje de piezas afectadas aumentaba (Gráfico Nº 3). 
Se aplicó la prueba estadística de Student para comparar la relación entre los valores obtenidos en los grupos de estudio antes y después del tratamiento. Al analizar los valores al 0,05\% de error se encontró que existía una varianza altamente significativa en los pacientes que respondieron adecuadamente al tratamiento (Tabla $\mathrm{N}^{\circ} 1$ ).

\section{Discusión}

El pH ha sido relacionado por mucho tiempo con la patología de la caries dental. Sin embargo, su papel en la patología en la enfermedad periodontal aún no está del todo claro. Muchos estudios han demostrado que el fluido crevicular se alcaliniza en pacientes con gingivitis y a medida que aumentaba la profundidad de la bolsa en pacientes con periodontitis. Como se sabe el líquido crevicular y sus diferentes productos drenan hacia la cavidad oral influyendo en la composición y propiedades de la saliva.

El presente trabajo de investigación trata de demostrar la relación existente entre el $\mathrm{pH}$ de la saliva total y la patología periodontal en sus dos principales tipos: gingivitis y periodontitis. Se postuló que el $\mathrm{pH}$ salival se encontraría alterado en la enfermedad periodontal a medida que aumentaba la progresión y severidad de la enfermedad. Asimismo se postuló que luego de un tratamiento exitoso que mejorará el control de placa, resolviera el sangrado al sondaje y la profundidad de bolsa, el $\mathrm{pH}$ salival debería disminuir. Al concluir el estudio se encontró que ambas premisas eran correctas, lo que fue puesto de manifiesto en varios hallazgos.

Uno de los principales datos que se obtuvieron demostró que en la enfermedad periodontal el $\mathrm{pH}$ salival se encuentra modificado. Es así que la media del $\mathrm{pH}$ salival encontrado en los pacientes con enfermedad periodontal se muestra más alcalino en relación a los pacientes del grupo control. En el grupo control se obtuvo un $\mathrm{pH}$ medio de 6,9, mientras que en pacientes con gingivitis y periodontitis fue de 7,3 y 7,9 respectivamente. Es así que también se puede ver que existe una marcada diferencia entre el $\mathrm{pH}$ salival de los pacientes con gingivitis $\mathrm{y}$ los que fueron diagnosticados con periodontitis.

$\mathrm{Al}$ evaluar la relación entre el $\mathrm{pH}$ salival y la severidad de la enfermedad periodontal encontramos una relación directamente proporcional. Es así que a medida que la enfermedad progresaba se observó una mayor alcalinización del $\mathrm{pH}$ de la saliva de los pacientes. Sin embargo, la mayor diferencia de $\mathrm{pH}$ salival se observo en el paso de gingivitis a periodontitis.

El otro hallazgo importante fue que los niveles de $\mathrm{pH}$ salival disminuyeron hasta niveles casi normales luego de realizado el tratamiento periodontal y cuando e paciente respondió favorablemente a éste. Asimismo, en los pacientes que no respondieron al tratamiento o lo hicieron deficientemente el pH salival se mantuvo o disminuyó levemente.

Por lo tanto, luego de analizar lo resultados obtenidos en el presente estudio, se puede decir que el $\mathrm{pH}$ salival tiene una relación directa con la enfermedad periodontal y qué éste aumenta a medida que progresa la enfermedad. Probablemente y con más estudios se logre establecer las escalas de $\mathrm{pH}$ salival propias de cada grado de avance de la enfermedad periodontal. Con estos conocimientos se podría usar la medición del $\mathrm{pH}$ salival como un medio diagnóstico y su modificación tal vez como un medio terapéutico.

\section{Conclusiones}

El pH salival presenta valores más alcalinos en pacientes con enfermedad periodontal en relación con pacientes periodontalmente sanos. Asimismo, los valores de $\mathrm{pH}$ salival se elevan más en la periodontitis que en la gingivitis. A medida que la progresión y la extensión de la enfermedad periodontal aumentan, el $\mathrm{pH}$ salival presenta una escala de mayor alcalinidad.

\section{Referencias Bibliográficas}

1. Arce, R. Terapia periodontal del futuro Colombia Médica Editora Médica del Valle 2004; Vol. 35(3) (Supl. 1)

2. Bascones, A., Bullón, A., Castillo J., Machucha, G., Manso, F., Serrano, J. Bases farmacológicas de la terapéutica odontológica. España: Ediciones Avances. 2000

3. Banderas J., Gonzáles, M., Sánchez M., Millán E., López, A. Flujo y concentración de proteínas en saliva total humana. Salud Pública de México 1997; Vol. 39(5)

4. Canales F., Alvarado E., Metodología de la investigación. 4a ed. Honduras: Editorial MS. 2000

5. Eggert F., Drewell L., Bigelow J., The $\mathrm{pH}$ of gingival crevices and periodontal pockets in children, teenagers and adults Oral biology 1991; 36(3)
6. Forscher B.K., Paulsen A.G., The pH of the periodontal pocket and the glycogen content of the adjacent tissue. Journal of Dental Research 1953; Vol. 33(4)

7. Fujikawa K., Numasaki H., Kobayashi M., $\mathrm{pH}$ determination in human crevicular fluids. Examination of the $\mathrm{pH}$ meter and evaluation of the correlation between $\mathrm{pH}$ level and clinical findings or the microflora in each periodontal pocket, Nippon Shishubyo Gakkai Kaishi 1989; 31(1)

8. Galgut P., The relevance of $\mathrm{pH}$ to gingivitis and periodontitis, Journal Academy periodontology 2001; 3(3)

9. Jenkins N., Fisiología y bioquímica bucal 2a ed. Reino Unido: Edit. Limusa. 1992

10. Kaufman, E., Lamster, I. Analysis of saliva for periodontal diagnosis. Journal of Clinical Periodontology 1998; Vol. 2: 453-465

11. Kobayashi K., Soeda W. , Watanabe T:, Gingival crevicular $\mathrm{pH}$ in experimental gingivitis and trauma oclusal in man, Journal of Periodontology 1998; Vol. 69 (9)

12. Lindhe J. Periodontología clínica e implantología odontológica 4 a ed. Madrid: Edit. Médica Panamericana. 2003

13. Navazesh M., Methods for collecting saliva, The Annals of New Cork Academy of Sciences 1993; Vol. 694

14. Ozmeric, N. Advances in periodontal disease markers. Clinica Chimica Acta 2004; 343: 1 - 16

15. Willians E., Bioquímica básica $y$ aplicada, 2a ed. México: El Manual Moderno. 1982

16. Sociedad Española de Periodoncia y Osteointegración. Manual SEPA de Periodoncia y terapéutica de implantes. Madrid: Edit. Médica Panamericana. 2005

17. Streckfus C., Bibler L. Saliva as a diagnosis fluid. Oral diseases 2002; Vol. 8: $69-76$

18. Watanabe T., Soeda W., Kobayashi K., Nagao M., The $\mathrm{pH}$ value changes in the periodontal pockets. Bull Tokyo Med Dent Univ. 1996; 43(4)

Recibido :11-08-2008

Aceptado para publicación: 15-09-2008 